\title{
Innovative concepts for aerodynamic control of wind turbine rotors
}

\section{P. Jamieson}

Garrad Hassan and Partners Ltd., 6.05 Kelvin, West of Scotland Science Park, Maryhill Road, Glasgow G20 0SP, Scotland, UK
A. Bowles
ETSU, UK
A. Derrick
NEL, Scotland, UK
W. Leithead
Strathclyde University, Strathclyde, Scotland, UK
and

M. Rogers
Industrial Systems and Control Ltd., Scotland, UK

\section{Summary}

New systems for the aerodynamic control of wind turbine rotors are being studied in various projects funded by the UK Department of Energy. Results from a current project, ongoing at the National Wind Turbine Test Centre (NWWTC) in Scotland are presented. These systems show the promise of much cheaper and more affective aclive control of horizontal axis wind turbines than has been achieved with full span and partial span pitching systems.

\section{Introduction}

New systems for overspeed protection and aerodynamic control of horizontal axis wind turbines are presented. They are in the general class of blade tip systems, but differ from the conventional rotating tip in two main respects.

The aerodynamic effectiveness of a tip system, as a brake, depends on the surfaces generating drag or negative lift. A large aerodynamic surface area can be utilised without involving the whole of structure in the tip area. The structural engineering of the tip system is then much easier.

Loads on a tip system fully deployed for maximum braking, preventing dangerous overspeed, must be reacted on the blade structure. In particular, for tip systems designed for active control the braking loads in overspeed dominate 
the structural requirements. The loads need not, however, react fully on the actuating system and unnecessary load demand and cost on the actuation system can be reduced.

Two main new systems are to be discussed, the SLEDGE (sliding leading edge system in which the leading edge of the aerofoil translates radially outward), and the FLEDGE (flying leading edge system in which the upper surface of the aerofoil is rotated). The UK Department of Energy (DEn) have supported the initial development of these concepts, Jamieson et al. [1]. Comparisons with conventional systems are made and progress of a project also funded by DEn and underway at the NWTC is discussed. The project at NWTC involves computer simulation of wind turbine systems with the new aerodynamic control devices, mechanical design of systems leading to construction of mechanisms for laboratory testing and recommendations for possible field testing of complete systems on a full scale wind turbine.

\section{The SLEDGE}

The idea is to achieve an effective aerodynamic regulation from a minimum of blade span and surface area in the blade tip region. A part of the leading edge slides out radially (Fig. 1). The main features are:

(a) fail-safe operation linked directly to centrifugal force,

(b) good aerodynamic performance due to the following three effects,

- destruction of the positive power production in the blade tip area,

- creation of addition drag in the blade tip area,

- displacement of the leading edge radially outward into an increased relative wind velocity, further augmented by rotating the leading edge to generate negative lift,

(c) small control surface area and hence potential for low cost.

The NWTC project involves the design of a sledge system which has a very low weight in the sliding part.

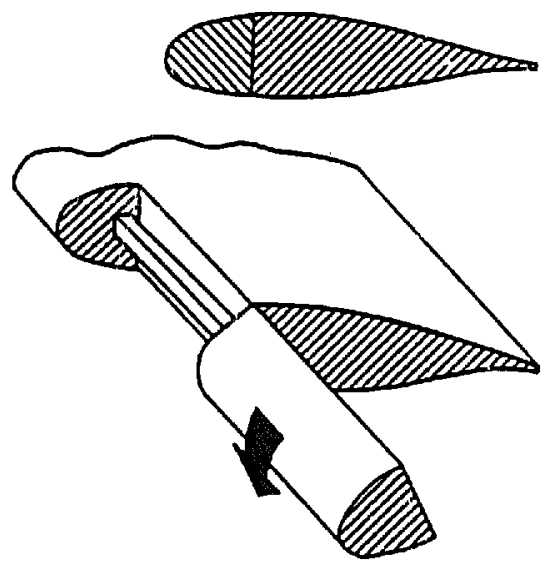

Fig. 1. 


\section{The FLEDGE}

This idea was conceived after wind tunnel tests Jamieson et al [1] of various SLEDGE sections at Imperial College, London. The striking features of these tests (see Fig. 2) were the high lift and stall delay developed by the leading edge part of the SLEDGE system.

The fact that such sections do develop high lift is well known to aerodynamicists, but not hitherto seen to be of practical use. This prompted the idea of purely rotating a part of the leading edge to use this high lift at negative incidence, exposing a high drag trailing section as with the SLEDGE. It was then considered that an extended upper surface plate could be included to create even more drag at full deployment. To some extent the fledge has then become quite like a conventional rotating tip. The differences in the structural engineering and actuation systems, however, are very significant.

Two types of fledge system are being studied in the NWTC project. These are termed respectively, the fledge with ball joint actuation, and the fledge with offset hinge-line.

The fledge with ball joint actuation (Fig. 3 ) is particularly conceived as an active control system (it should be stressed that the figures of SLEDGE and FLEDGE systems are intended to be clear schematics of operating principles, they do not reflect the actual structures or the latest mechanisms under present development.

Features of this system are the use of the inevitable lift and suction on the upper surface of the aerofoil to assist in starting the deployment of the device, with subsequent damping of the motion from the high drag developing as the plate rotates to full deployment at $90^{\circ}$. A further feature is that through an increasing mechanical advantage on the actuating arm, most of the high drag braking force at full deployment is reacted on the structure and not on the sliding mass actuator. The deployment is, therefore, smooth, the actuating mass is small (about $5 \mathrm{~kg}$ for a $33 \mathrm{~m}$ diameter rotor system) and the system is

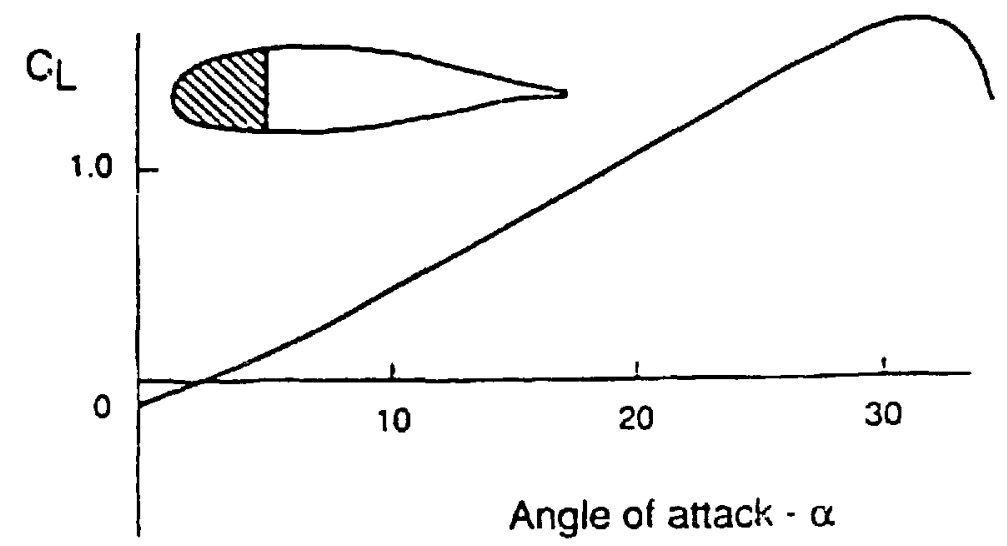

Fig. 2. 

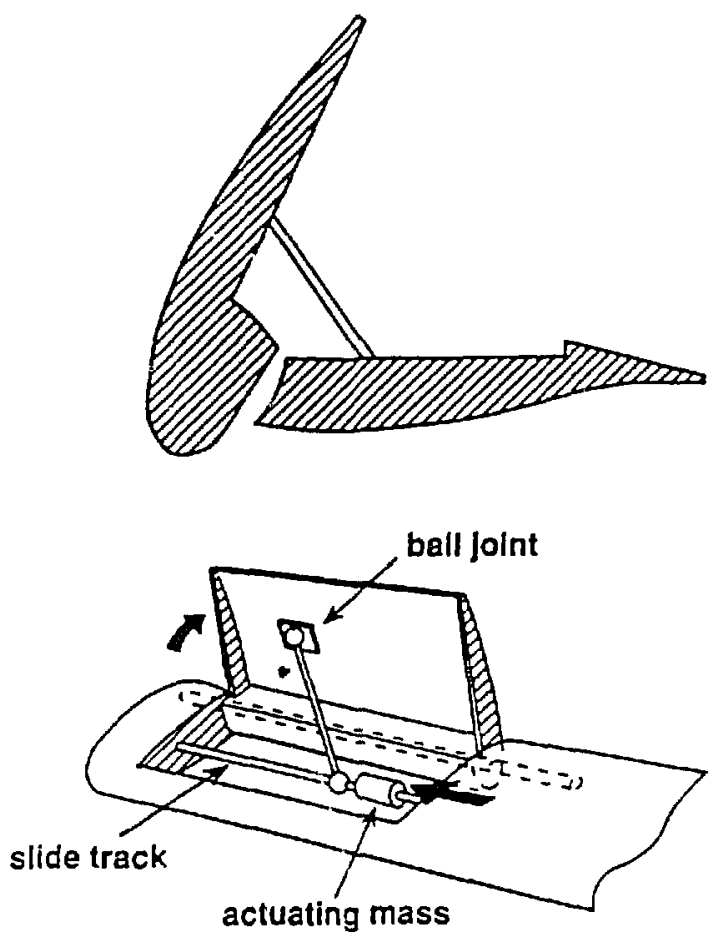

Fig. 3.

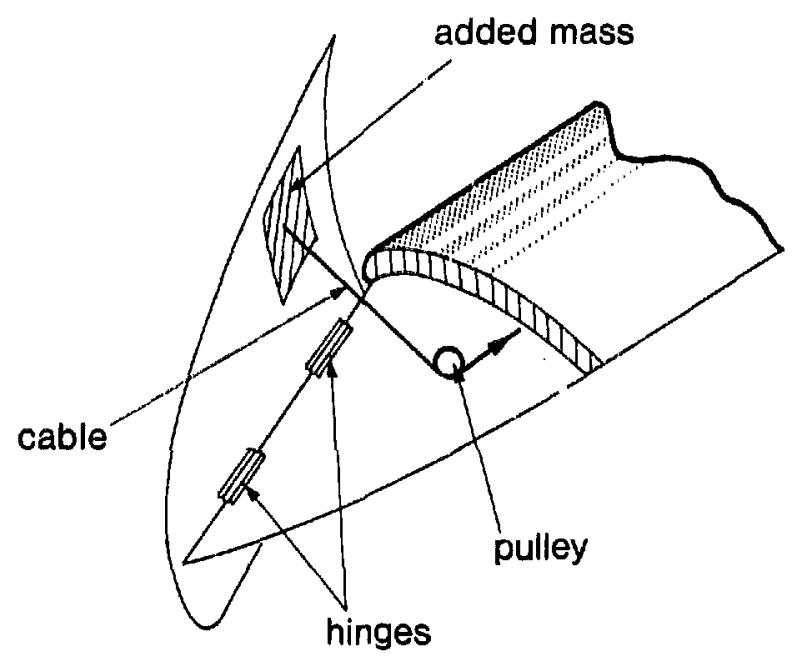

Fig. 4.

controlled via lightly loaded cables from an actuator in the hub area. Very little damping is needed, but a fast response is observed (deployment times of the order of 0.2 to $0.5 \mathrm{~s}$ ).

The fledge with offset hinge-line (Fig. 4), is primarily designed as a low-cost air brake, say, for example, for a stall regulated rotor. The idea is that the line of rotation of the fledge is swept back at about $20^{\circ}$ to the radial direction. A mass (about $20 \mathrm{~kg}$ for a $33 \mathrm{~m}$ rotor system with a $2 \mathrm{~m}$ long fledge plate) is added 
in the trailing edge of the plate. It will deploy under the action of centrifugal force with a cable to restrain the motion acceptably. This appears to be a particularly simple system, although the positive opening and possible subsequent flapping of the fledge plate are of concern and further mechanisms may be needed in the system.

\section{Comparisons with conventional systems}

A criterion for overspeed protection and the basis of effective control devices are discussed in Leithead et al. [3]. Aerodynamic analyses of various systems based on published aerodynamic data allows a determination of the minimum span-wise extent of various types of system that may be used for control or aerodynamic braking. Results for a $45 \mathrm{~m}$ diameter rotor similar to the Howden HWP 750/45 are presented in Fig. 5. The shaded area represents the amount
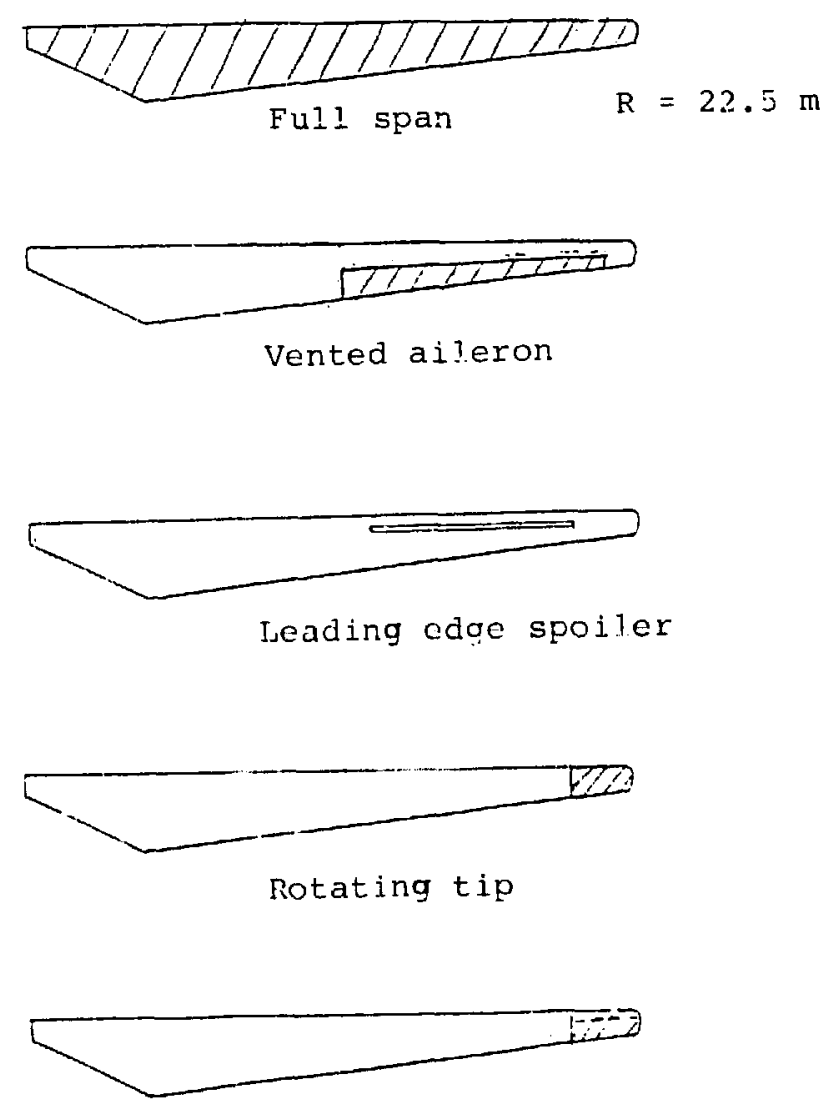

FLEDGE

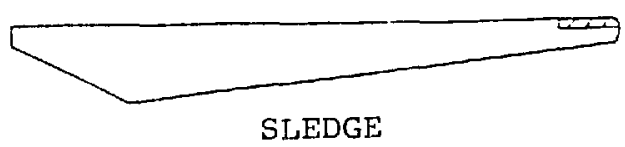

Fig. 5. 
TABLE 1

\begin{tabular}{lll}
\hline & Wind turbine system & Actuator design load (kN) \\
\hline 1 & $\begin{array}{l}\text { Standard Howden } 750 / 45 \text {, operating } \\
\text { wind turbine }\end{array}$ & 70 \\
$\begin{array}{l}\text { Same turbine with a fledge system } \\
\text { (ball joint actuated type) }\end{array}$ & 7 \\
\hline
\end{tabular}

of articulated blade surface. In each case, the amount of span is the minimum necessary to meet the Risø criterion for air brakes, (that the rotor should not idle off-load in a wind of $30 \mathrm{~m} / \mathrm{s}$ at a speed greater than its normal operating speed) except in the case of full span pitching where the whole blade is moved as a matter of choice.

Only the latter three devices, the conventional rotating tip, fledge and sledge are compact short span devices. In the next stage of comparing actuator loads the decisive advantages of the new systems are apparent (Table 1).

In addition to the great reduction in actuation loads, it is likely that a mechanically simpler and economic blade tip structures will be possible. The system depicted in Fig. 4 has been elegantly engineered by the Wind Energy Group for a thin highly elliptical tip in wood and glass with no metallic components embedded for structural support. Nevertheless, the metallic components (such as hinges, actuating weight and pulley) are supported by the composite structure.

\section{Simulation studies}

Computer simulations of the operational behaviour of SLEDGE and FLEDGE systems under study in the NW'TC project, have been invaluable in guiding the dynamic design of systems and alerting the investigator to unexpected modes of behaviour. The simulation models use the ACSL (Advanced Continuous Simulation Language) package which automatically generates FORTRAN code to solve systems of non-linear differential equations. Well validated models of the drive train and control systems of various operating wind turbines have been developed in previous DEn funded work. These projects were aimed at investigating and improving active control of wind turbines.

Figure 6 illustrates the behaviour of an air brake system for a three bladed rotor using the fledge with offset hinge-line (as in Fig. 4). At time $t=0$, the grid is disconnected and the rotor starts to overspeed. The three plates deploy rapidly when approximately $10 \%$ overspeed has been reached to $\theta=90^{\circ}$. The rotor angular speed $(w)$ then reduces, but due to the effects of turbulence, the plates start to flap. The simulation can show the sensitivity of the flap behaviour to turbulence intensity and pitching moment characteristics. 

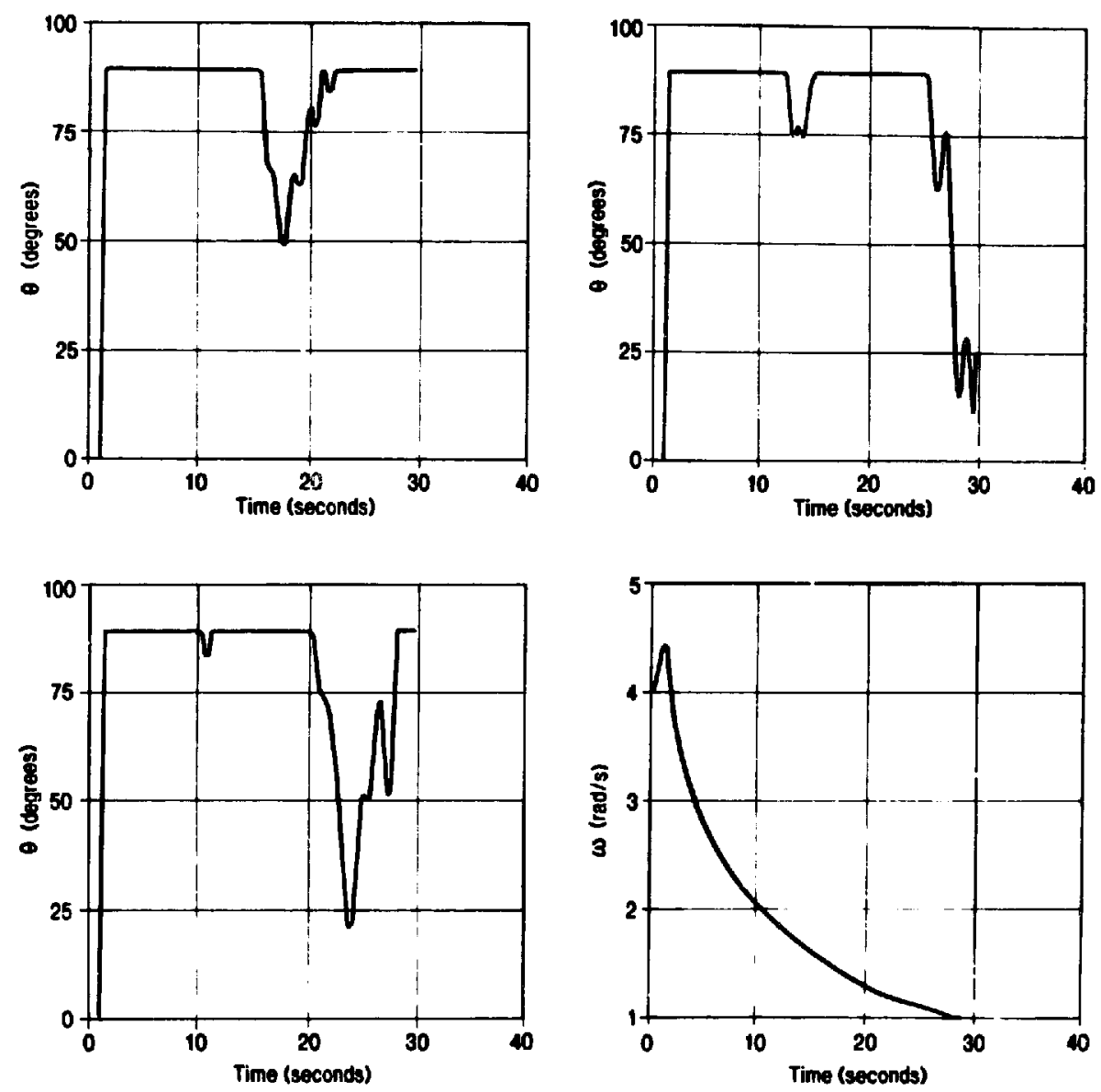

Fig. 6.
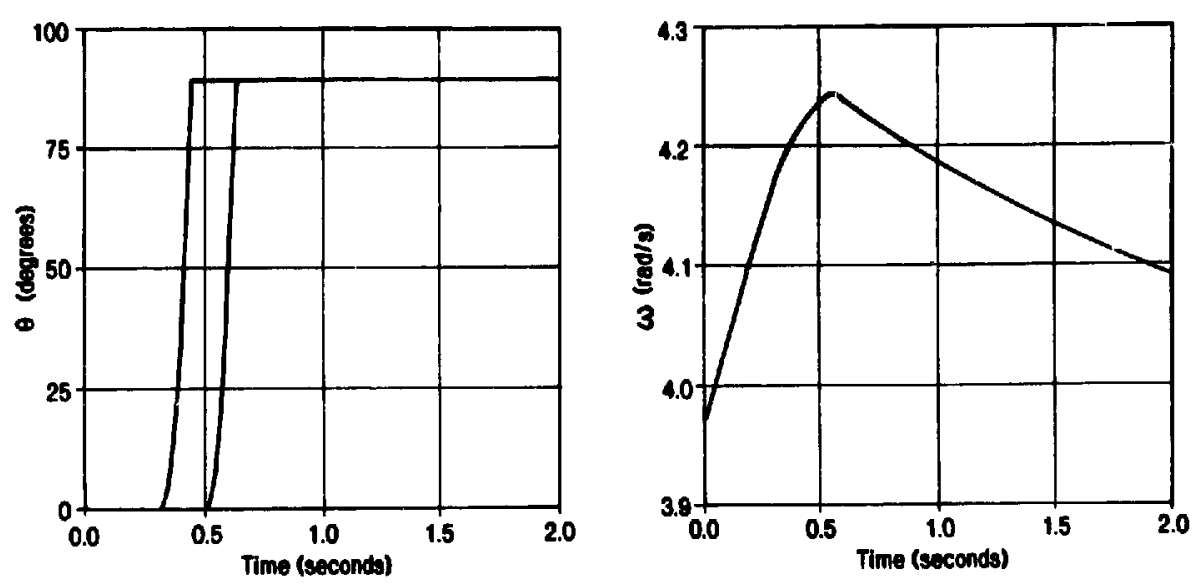

Fig. 7.

Air brakes have been designed with no connection or coordination between each blade tip, for example the Aerostar. It is, therefore, possible for only one tip to deploy and thereby slow the rotor, reducing the activating centrifugal force so that the other tips do not deploy. This is illustrated in Fig. 7, for a 
system with ball joint type fledges, as depicted in Fig. 3, where the release of each fledge is controlled locally by centrifugal catches and there is no cable system to the hub enforcing coordination.

In a wind of $11 \mathrm{~m} / \mathrm{s}$ only 2 of the 3 fledges successfully deploy. Interestingly, (see Fig. 8), if some delay damping is introduced (i.e. each tip has a small shock absorber in the system) thus preventing too early a deployment of the first 2 tins the third tip is triggered and all 3 deploy. Nevertheless, in the limit of the iowest operating windspeeds around $6 \mathrm{~m} / \mathrm{s}$, it will not be possible to guarantee, by this type of design, that all fledges will deploy.

A coordinated cable system is, therefore, preferred and does not appear to be a great added cost.

Moreover, the preferred kind of balljoint actuated fledge system involves cabling to coordinate the fledges and seems very suitable for active control. The fledges have low inertia and are very responsive, keeping the actuator demand low (for example $2 \mathrm{kN}$ for the load to operate all three cables of the $33 \mathrm{~m}$ rotor system ). The simulation is used to tune system characteristics and to provide load specification for design of the actuation system and local structural connections.

A typical system for a $33 \mathrm{~m}$ rotor operating in the design case of a $30 \mathrm{~m} / \mathrm{s}$ wind with loss of electrical load is presented in Fig. 9. During the deployment of the fledges, the peak damping force on the hub actuator (operating on three connected cables, one from each blade) is $750 \mathrm{~N}$ and the cable tension (see Fig. 9) does not excee the load of $300 \mathrm{~N}$, which is applied to keep the fledge plate positively closed in normal operation. The highest load in the system is a side reaction peaking at $5 \mathrm{kN}$, which is translated onto the blade structure.

Considering the actuating system, an applied force of $1.8 \mathrm{kN}$ due to centrifugal force on the actuating mass is overcoming a peak friction force of $400 \mathrm{~N}$. Resistance from the cable tension is less than $100 \mathrm{~N}$ after the deployment is in progress. Compared to the massive loadings inherent in full span pitch ar-
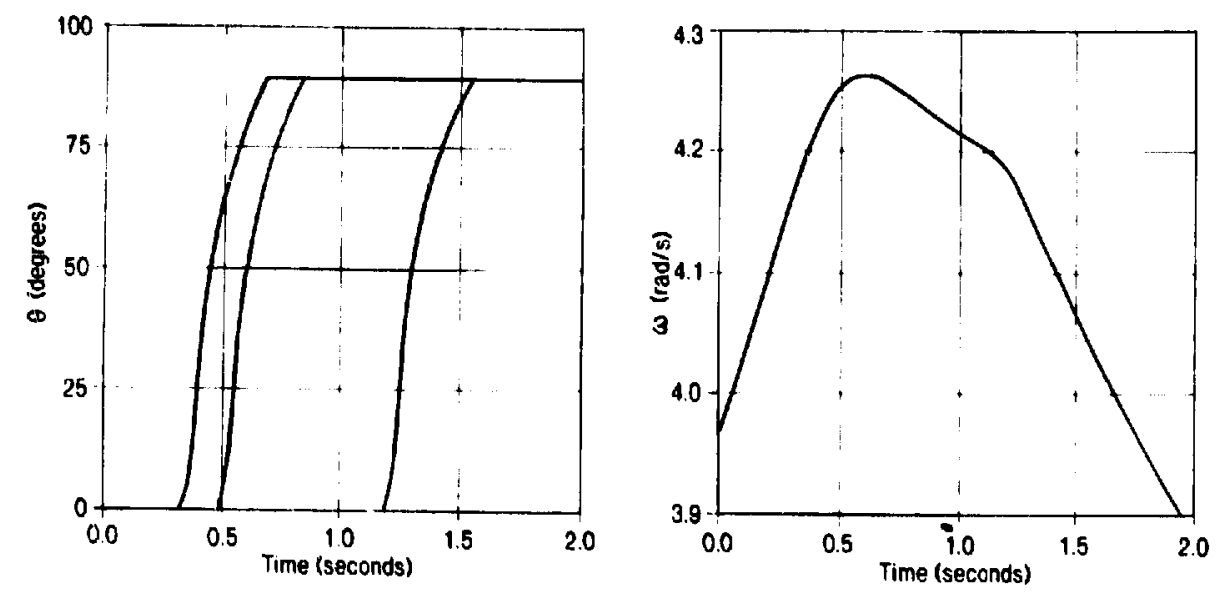

Fig. 8. 

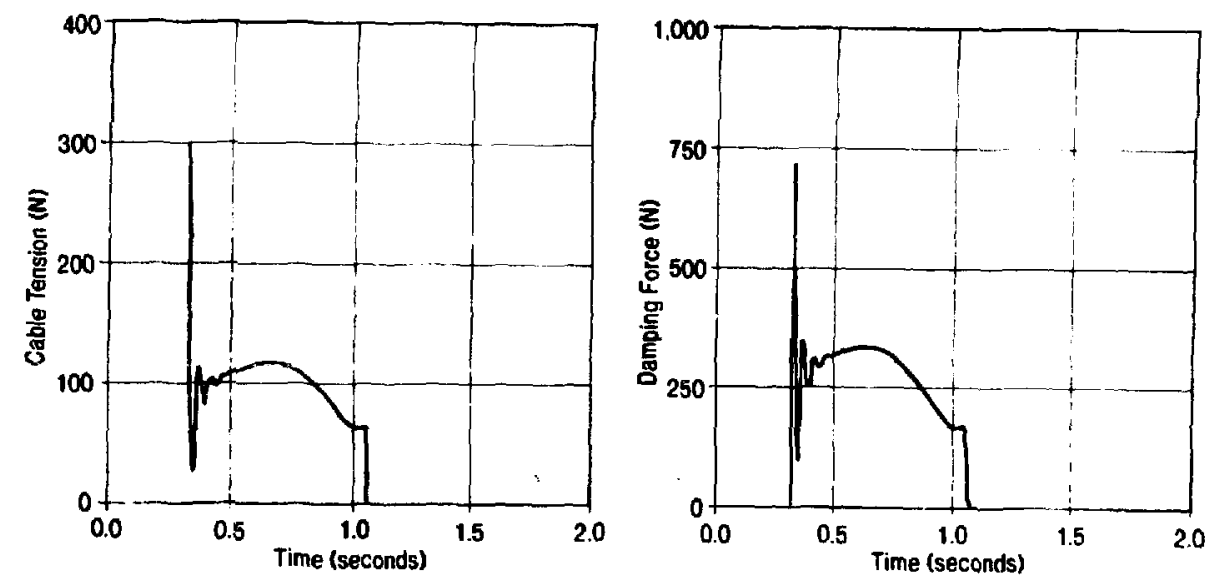

Fig. 9.

\section{POWER REGULATION USING 1.5m FLEDGE}

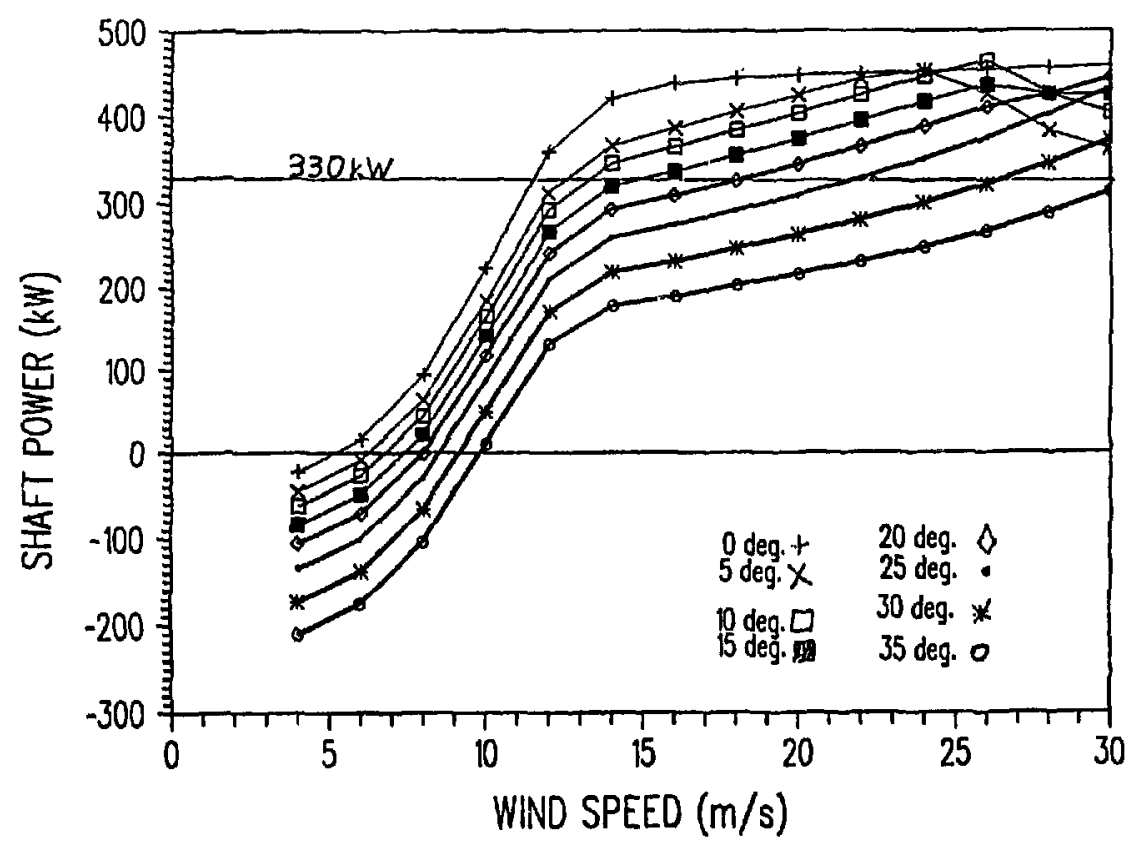

Fig. 10.

rangements and the substantial loads ( 10 to $30 \mathrm{kN}$ typically) involved in conventional rotating tip systems, the potential for the new systems to be very economic should be evident.

The fledge system (and also the sledge) have low inertia and good control characteristics. Steady state power performance predictions for various angles of deployment of a fledge are presented in Fig. 10.

A control system is being designed using the general classical control methods (i.e. not specifically PID control) under development by Leithead et al. [3]. 


\section{Conclusions}

Suitably designed blade tip systems can provide low cost aerodynamic braking superior to the historic rotating tip systems where the whole tip structure is overhung.

A striking feature of the new systems is that for very little added cost, active control can be provided from actuating systems in the hub or nacells area, avoiding complication in the blade tips.

\section{Acknowledgements}

This work was carried out as part of the UK Department of Energy Wind Energy Programme.

\section{References}

1 P. Jamieson and P. Agius, SLEDGE - a novel braking device for HAWTs. For DEn, E/5A/ 5119/1989. Mar. 1989.

2 P. Jamieson and P. Agius, A comparison of aerodynamic devices for control and overspeed protection of HAWTs. BWEA 12, Norwich, March 1990.

3 W. Leithead, S. De la Salle and D. Reardon, Classical design of a pitch control system. BWEA 12, Norwich, March, 1990. 